\title{
Growing tumors induce a local STING dependent type I IFN response in dendritic cells.
}

\author{
Lisa Andzinski ${ }^{1}$, Julia Spanier ${ }^{2}$, Nadine Kasnitz ${ }^{1}$, Andrea Kröger ${ }^{3,4}$, Lei Jin ${ }^{5}$, Melanie M. Brinkmann ${ }^{6,7}$, \\ Ulrich Kalinke $^{2}$, Siegfried Weiss ${ }^{1,8}$, Jadwiga Jablonska ${ }^{1,9^{*}}$ and Stefan Lienenklaus ${ }^{1,2,10^{*}}$ \\ ${ }^{1}$ Molecular Immunology, Helmholtz Centre for Infection Research, Braunschweig, Germany \\ ${ }^{2}$ Institute for Experimental Infection Research, Twincore, Centre for Experimental and Clinical Infection Research, a \\ joint venture between the Helmholtz Centre for Infection Research, Braunschweig, and the Hannover Medical \\ School, Hannover, Germany \\ ${ }^{3}$ Innate Immunity and Infection, Helmholtz Centre for Infection Research, Braunschweig, Germany \\ ${ }^{4}$ Institute of Medical Microbiology, Otto-von-Guericke-University, Magdeburg, Germany \\ ${ }^{5}$ Center for Immunology and Microbial Disease, Albany Medical College, Albany, USA \\ ${ }^{6}$ Viral Immune Modulation, Helmholtz Centre for Infection Research, Braunschweig, Germany \\ ${ }^{7}$ Institute of Virology, Hannover Medical School, Hannover, Germany \\ ${ }^{8}$ Institute of Immunology, Hannover Medical School, Hannover, Germany \\ ${ }^{9}$ Translational Oncology, Department of Otorhinolaryngology, University Hospital, University of Duisburg-Essen, \\ Essen, Germany \\ ${ }^{10}$ Institute for Laboratory Animal Science, Hannover Medical School, Hannover, Germany \\ * Both authors contributed equally to this work
}

Address correspondence to: Stefan Lienenklaus, ZTL Imaging-Center, Hannover Medical School, Carl-Neuberg-Str. 1, 30625 Hannover, Germany; Phone: + 49511532 7385; E-mail: lienenklaus.stefan@mh-hannover.de or Jadwiga Jablonska, Translational Oncology, Department of Otorhinolaryngology, University Hospital, University of Duisburg-Essen, Hufelandstr. 55, 45147 Essen, Germany; Phone: + 49201723 3190; E-mail: jadwiga.jablonska@uk-essen.de

In this study we visualize IFN- $\beta$ expression during tumor growth in a sensitive in vivo reporter system. We identify dendritic cells as major cellular source of type I IFN in tumors and demonstrate that STING and the downstream transcription factors IRF3 and IRF5 are crucial for IFN induction. The characterization of the TypeI IFN induction pathway significantly adds to the current knowledge about the involvement of TypeI IFNs in tumor development. 


\begin{abstract}
The importance of endogenous type I IFNs in cancer immune surveillance is well established by now. Their role in polarization of tumor-associated neutrophilic granulocytes into anti-tumor effector cells has been recently demonstrated. Yet, the cellular source of type I IFNs as well as the mode of induction is not clearly defined. Here, we demonstrate that IFN- $\beta$ is induced by growing murine tumors. Induction is mainly mediated via STING-dependent signaling pathways, suggesting tumor derived DNA as trigger. Transcription factors IRF3 and IRF5 were activated under these conditions which is consistent with tumor infiltrating dendritic cells (DCs) being the major cellular source of IFN- $\beta$ at the tumor site. Besides DCs, tumor cells themselves are induced to contribute to the production of IFN- $\beta$. Taken together, our data provide further information on immune surveillance by type I IFNs and suggest novel potent cellular targets for future cancer therapy.
\end{abstract}




\section{Introduction}

Type I interferons (IFN) were discovered more than fifty years ago as factors responsible for interference with viral infections. ${ }^{1}$ By now, it is known that in humans and mice the type I IFN family comprises at least 12 IFN- $\alpha$ subtypes, IFN- $\beta$, IFN- $\varepsilon$ and IFN- $-{ }^{2}$ All of them signal via a common receptor (IFNAR), induce the expression of several hundred interferon inducible genes (ISGs) and have a broad range of biological functions. ${ }^{2}$ Within the type I IFNs, IFN- $\alpha$ and IFN- $\beta$ are characterized best. Besides their role in anti-viral and anti-microbial responses, they shape innate and adaptive immunity ${ }^{3}$, influence the maintenance of cellular homeostasis ${ }^{4}$, hematopoiesis ${ }^{5}$ and lymphocyte development ${ }^{6}$. In addition, type I IFNs show strong anti-tumor activity. ${ }^{7,8}$ The mechanisms of how type I IFNs contribute to immune surveillance against tumors are not fully understood, notwithstanding their beneficial effects in cancer therapy. ${ }^{7}$

Recently, we were able to demonstrate that type I IFNs, especially IFN- $\beta$, are major players in cancer immune surveillance and inhibit tumor-angiogenesis. We could show that IFN- $\beta$ interferes with the accumulation of pro-angiogenic tumor associated neutrophils (TANs). Massive enrichment of such cells took place in tumors of mice that lacked endogenous IFN- $\beta$. This drastically improved tumor angiogenesis. ${ }^{8}$ The induction of TANs towards a pro-angiogenic phenotype were observed under these circumstances. In addition, auto-attraction of TANs was up-regulated in absence of IFN- $\beta$ by influencing the expression of chemo attractants CXCL1, 2 and 5 as well as their receptor CXCR2. ${ }^{9}$ Moreover, increased longevity of TANs was observed in the absence of endogenous IFN- $\beta .{ }^{10}$ Finally, the formation of the pre-metastatic niche was improved when endogenous IFN signaling was suppressed resulting in massively enhanced metastasis formation. ${ }^{11}$ 
This raised the question whether such IFN effects are due to low levels of constitutive IFN- $\beta$ expression that we had observed before. ${ }^{12}$ Alternatively, an inflammatory trigger might be elicited by the tumor that induces type I IFN.

Signaling pathways leading to the induction of type I IFNs differ depending on the stimulus and the responding cell type. ${ }^{13}$ In this context a broad variety of danger-associated molecular patterns (DAMPs) and pathogen-associated molecular patterns (PAMPs) can be sensed via Toll-like receptors (TLRs), RIG-I-like receptors (RLRs) or cytosolic DNA receptors. This ultimately leads to the induction of type I IFNs via the TANK-binding kinase 1 (TBK1) and IFN regulatory factors 3 (IRF3) and IRF7. ${ }^{3}$ Besides IRF3 and 7, additional IRFs might be involved in type I IFN induction and regulation. For instance, IRF5 is crucial for IFN expression in myeloid dendritic cells. ${ }^{14}$ Apparently, the activation of IRF5 is cell type specific and recently multiple IRF5 splice variants with differential cellular localization and regulation have been identified. ${ }^{15}$

Here we demonstrate the induction of type I IFNs by different murine tumors using a sensitive mouse reporter system that previously unambiguously revealed the low constitutive IFN- $\beta$ production. ${ }^{12}$ Induction of IFN- $\beta$ in tumors was independent of TLR signaling. It was mediated via the adaptor protein of cytosolic DNA receptors STING, and, to a minor degree, the RLR adaptor Cardif in an IRF3/IRF5 dependent manner. As major producing cells tumor infiltrating dendritic cells were defined. 


\section{Materials and Methods}

\section{Animals}

All experiments performed in wild type animals were conducted using 8 to 12 week old female BALB/c (Harlan) or C57BL/6 (HZI) mice. The transgenic animals were bred in the animal facility of the Helmholtz Center for Infection Research (Braunschweig, Germany). BALB/c IFN$\beta^{+/ \Delta \beta-\text { luc }}\left(\operatorname{Ifnb} 1^{\text {tm1.2Lien }}\right)$, albino $\left(\mathrm{Tyr}^{\mathrm{c} 2 \mathrm{~J}}\right) \mathrm{C} 57 \mathrm{BL} / 6 \mathrm{IFN}-\beta^{+/ \Delta \beta-\text { luc }}\left(\mathrm{Ifnb} 1^{\mathrm{tm} 2.2 \mathrm{Lien}}\right), \mathrm{C} 57 \mathrm{BL} / 6$ IFN- $\beta^{+/ \text {flox} \beta-\text { luc }}$ $\left(\right.$ Ifnb1 $\left.1^{\text {tm2.1Lien }}\right) \times$ LysM cre $\left(\right.$ Lyz2 $\left.2^{\text {tm1(cre)Ifo }}\right)$, IFN- $\beta^{+/ \text {flox } \beta-l u c} \times$ CD11c cre (Tg(Itgax-cre) $1-1$ Reiz $)$, IFN$\beta^{+/ \text {flox } \beta \text {-luc }} \times$ CD4 cre $(\operatorname{Tg}(\mathrm{CD} 4-\mathrm{cre}) 1 \mathrm{Cwi}), \operatorname{IFN}-\beta^{+/ \text {flox} \beta \text {-luc }}$ x $\mathrm{CD} 19$ cre $\left(\mathrm{Cd} 19^{\mathrm{tm} 1(\mathrm{cre}) \operatorname{Cgn}}\right)$ have been previously described. ${ }^{16}$ In addition IFN- $\beta^{+/ f l o x \beta-l u c} x$ Tie 2 cre $(\operatorname{Tg}($ Tek-Cre $) 1$ Arnd $)$ were generated using the described breeding strategy. ${ }^{16} \mathrm{IRF}^{-/-}\left(\operatorname{Irf} 3^{\mathrm{tm} 1 \mathrm{Ttg}}\right), \operatorname{IRF}^{-/-}\left(\operatorname{Irf} 5^{\mathrm{tm} 1 \mathrm{Mak}}\right), \mathrm{IRF}^{-/-}\left(\operatorname{Irf} 7^{\mathrm{tm} 1 \mathrm{Ttg}}\right)$,

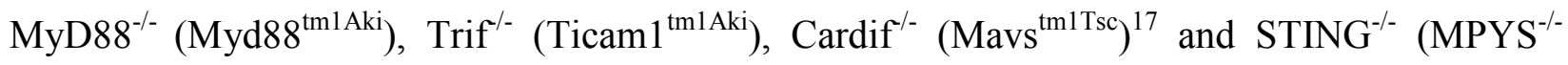
$/$ Tmem $\left.173^{\text {tm1Camb }}\right)^{18}$ IFN- $\beta$ reporter mice were generated by intercrossing the respective recombinant mice with IFN- $\beta^{\Delta \beta-l u c}$ mice on C57BL/6 background. An independent STING defective mutant (C57BL/6J-Tmem173 $\left.{ }^{\text {gt }} / \mathrm{J}\right)$ was additionally included. All mice were kept under specific pathogen free (SPF) conditions. The studies have been reviewed and approved by an ethic committee of the regulatory authorities LAVES of Lower Saxony (33.9-42502-04-13/1122 and $14 / 1455$ permission numbers).

\section{Tumor cell lines and transplantation}

B16F10 (C57BL/6), MCA205 (C57BL/6), 4T1 (BALB/c) or CT26 (BALB/c) tumor cells were cultivated in Iscove's Modified Dulbecco's Medium (IMDM) (Gibco BRL; Invitrogen) supplemented with $10 \%(\mathrm{v} / \mathrm{v})$ heat-inactivated fetal calf serum (FCS) (Integro), $250 \mu \mathrm{mol} / 1 \beta$ mercaptoethanol (Serva), and $1 \%$ (v/v) penicillin/streptomycin (Sigma-Aldrich). Cells were grown in monolayer at $37{ }^{\circ} \mathrm{C}$ in a humidified $\mathrm{CO}_{2}$ incubator. For transplantation, cells were 
harvested, washed and $1 \times 10^{5}$ cells were subcutaneously injected into the flank of mice. MCA205 fibrosarcoma was kindly provided by T. Blankenstein (Max Delbrück Center for Molecular Medicine, Berlin, Germany). Other tumor cell lines were purchased from ATCC. Tumor growth was monitored by measuring the length and width of the tumors using a caliper. Tumor volume was calculated assuming an ellipsoid shape (4/3pi*length* width $\left.{ }^{2} / 8\right)$.

\section{Detection of luciferase activity in vivo and ex vivo}

For luciferase activity assays from tissue, tissue fragments were homogenized in volumes of Reporter Lysis Buffer that were proportional to their weight (Promega). Lysates were mixed with LARII (Promega) and measured in a luminometer (Berthold). For noninvasive in vivo imaging, mice were injected i.v. with $150 \mathrm{mg} / \mathrm{kg}$ of D-luciferin in PBS (Perkin Elmer), anesthetized using Isofluran (Baxter) and monitored using an IVIS 200 imaging system (Perkin Elmer). Photon flux was quantified using the Living Image 4.3 software (Perkin Elmer).

\section{Cell preparation and fluorescence activated cell sorting (FACS)}

To assess the IFN- $\beta$ expression on cellular level, DCs or B cells from tumor and spleen were sorted. To this end, non-necrotic tumor tissue was cut into 1-2 $\mathrm{mm}^{3}$ pieces. The pieces were rinsed twice with PBS and digested using dispase/collagenaseA/DNase $(0.2 \mathrm{mg} / \mathrm{mL} 0.2 \mathrm{mg} / \mathrm{mL}$ $100 \mathrm{mg} / \mathrm{mL}$ ) in IMDM for $45 \mathrm{~min}$ at $37^{\circ} \mathrm{C}$. Cell suspensions and remaining debris were then pushed through $50 \mu \mathrm{m}$ disposable filters (Cell Trics, Partec). Cells from spleens were flushed out using $2 \mathrm{ml}$ IMDM and filtered through $50 \mu \mathrm{m}$ disposable filters. Subsequently, erythrocytes were removed using erythrocyte lysis buffer (ACK buffer). To avoid unspecific binding of the antibodies, cells were treated with anti-mouse CD16/CD32 Fc block. Single cell suspensions were stained. CD $11 \mathrm{~b}^{+} \mathrm{CD} 11 \mathrm{c}^{+} \mathrm{DCs}, \mathrm{CD} 11 \mathrm{~b}^{-} \mathrm{CD} 11 \mathrm{c}^{+} \mathrm{DCs}$ and $\mathrm{CD} 19^{+} \mathrm{B}$ cells were sorted using a FACSAria $^{\mathrm{TM}}$ cell sorter (BD Bioscience) and the purity of cells was confirmed by reanalysis. 


\section{RNA isolation for PCR analysis}

Cells/tissues were harvested and total RNA was extracted using the RNeasy Kit (Qiagen) according to the manufacturer's protocol. DNA contamination in total RNA was eliminated by incubation with DNaseI (Amersham Pharmacia Biotech) and cDNA was prepared using RevertAid First Strand cDNA Synthesis Kit (Fermentas) and oligo(dT) as primers.

To investigate the expression of Ifnbl, qRT-PCR was performed using Power SYBR Green Master Mix (Applied Biosystems) and analyzed on a 7500 Real Time PCR System (Applied Biosystems) or LightCycler 480 II (Roche) respectively. Real-Time RT-PCRs were performed with the following primers: Ifnb1: (s) 5'- CTG GCT TCC ATC ATG AAC AA-3'; (as) 5' - CAT TTC CGA ATG TTC GTC CT-3'.To standardize the cDNA, the house keeping gene Rps 9 was used with primer pairs: (s) 5'-TTG ACG CTA GAC GAG AAG GAT-3'; (as) 5'-AAT CCA GCT TCA TCT TGC CCT-3'.

\section{Statistics}

Statistical analyses were performed using a 1-tailed Student's $t$ test for single-value comparisons and one-way ANOVA to compare three or more different data sets. $P<0.05$ was considered significant. Values are expressed as mean \pm SEM. 


\section{Results}

\section{IFN- $\beta$ is induced in transplantable murine tumors}

Type I IFN production can be triggered by a broad variety of molecules during infection and inflammation. It is induced via several pathways and produced by virtually all types of cells. ${ }^{19}$ We had shown before that IFN- $\beta$ is a crucial factor in immune surveillance of cancer. Thus, the question arose whether IFN- $\beta$ is specifically induced by the tumors or whether the IFN- $\beta$ effect is due to constitutively expressed IFN- $\beta$. We also wanted to define the signaling pathways and the producer cell types. Thus, we made use of the sensitive murine IFN- $\beta$ reporter system recently introduced by us. ${ }^{12}$ In this mouse line, one IFN- $\beta$ coding allele was replaced by the firefly luciferase gene with the promoter remaining unaltered. The other allele was not changed to allow type I IFN responses.

Syngeneic 4T1 mammary carcinoma, CT26 colon carcinoma, MCA205 fibro sarcoma and LLC1 lung carcinoma cells were subcutaneously (s.c.) transplanted onto the back of BALB/c or albino C57BL/6 IFN- $\beta$ reporter mice, respectively. IFN- $\beta$ induction was followed by noninvasive optical in vivo imaging over 14 days, as depicted in Figure 1. Of note, under these conditions only host cells are capable of producing luciferase. Therefore, a bioluminescent signal in the tumor indicated expression of IFN- $\beta$ by tumor invading host cells.

One day after tumor injection, an elevated signal was detected at the inoculation site in all mice independent of the kind of tumor (Fig. 1). This elevated signal was absent in untreated as well as PBS-injected control mice (data not shown). By 72 hours after tumor cell injection, the signal decreased to background levels. At that time, tumors were neither visible nor palpable. As the tumors started to become palpable, the luciferase signal became detectable again. On day 14 after 
inoculation, the tumors were well established and showed elevated reporter signals as observed initially after injection. This indicates an inflammatory response of host cells in the growing tumors and expression of IFN- $\beta$.

To quantitatively compare levels of IFN- $\beta$ expression in the different tumors with constitutive IFN- $\beta$ expression in various host tissues, we inoculated reporter mice with MCA205 or CT26 tumor cells. On day 14, the mice were sacrificed and homogenates of tumor tissue, lung, spleen and thymus were tested for enzymatic activity of luciferase. We had previously determined constitutive IFN expression in different host tissues and found little background activity in tissues like lung and muscle, but elevated levels in spleen, lymph nodes and liver. Particular high expression was found in the thymus. Interestingly, high thymic IFN- $\beta$ expression was dependent on the transcription factor AIRE. ${ }^{12}$

The luciferase signal detected in the tumor samples of MCA205 and CT26 tumors was significantly higher compared to spleen and reached or exceeded the intensity of IFN- $\beta$ reporter activity in the thymus (Fig. 2). These results indicate that invading host cells in a growing tumor produce relatively high amounts of IFN- $\beta$.

\section{DCs are producers of IFN- $\beta$ in solid tumors}

To characterize the host cell population responsible for IFN- $\beta$ production in growing tumors, we made use of IFN- $\beta^{\text {flox } \beta \text {-luc }}$ conditional reporter mice. ${ }^{12,16}$ In these mice the luciferase reporter function is dependent on tissue specific cre expression. Tie2 Cre, CD19 Cre, CD4 Cre, LysM Cre and CD11c Cre were crossed into such mice to specifically detect IFN- $\beta$ production in hematopoietic and endothelial cells, B cells, T cells, myeloid cells and DCs, respectively, via ex vivo luciferase measurements. 
MCA205 tumors were grown in such mice and analyzed on day 14 after inoculation. In homogenates of tumors from Tie2, LysM and CD11c specific IFN- $\beta$ reporter mice, signal intensity correlated to $100 \%$ with signal strength observed for the global reporter animals (Fig. 3 A to C). This indicates that a cell population derived from hematopoietic Tie2 expressing precursor cells is responsible for IFN- $\beta$ production in growing tumors. In addition, such cells express or expressed the myeloid lineage marker LysM and the integrin CD11c. Interestingly, at least part of the tumor-associated host cells that produce IFN- $\beta$ in these murine fibrosarcomas had once expressed or still express CD4 (Fig. 3 D). No signal could be detected in CD19 specific reporter mice (Fig. $3 \mathrm{E}$ ), indicating that B cells do not contribute to IFN- $\beta$ production in growing tumors. DCs express CD11c, are of hematopoietic origin, and could be derived from myeloid precursor cells. They match the criteria defined by the cell specific reporters. Therefore DCs are most likely the cells producing most of type I IFN in solid tumors. Consistently, DCs are the major source of type I IFNs under many circumstances and were demonstrated to produce IFN- $\beta$ in tumor draining lymph nodes. ${ }^{20}$

To validate these findings, DC populations were sorted from tumors of C57BL/6 mice and tested for expression of IFN- $\beta$ (Fig. 3 F). Using splenic DC populations and CD19 ${ }^{+}$B cells as control, tumor-associated DC populations i.e. CD1 $1 b^{+}$DCs could be shown to express high amounts of IFN- $\beta$. Therefore they are indeed the major cellular source of type I IFN in growing tumors.

\section{STING dependent signaling is responsible for IFN- $\beta$ induction in growing tumors}

Three major classes of pattern recognition receptors (PRRs) are known to mediate type I IFN induction upon ligand engagement. TLRs sense a broad spectrum of PAMPs and DAMPs and signal via the adaptor proteins Trif and MyD88. ${ }^{21}$ RLRs are cytosolic RNA receptors that signal via the adaptor protein Cardif/IPS-1/MAVS to induce type I IFN expression. ${ }^{22}$ The third receptor 
system capable to mount type I IFN responses are cytosolic DNA receptors. They sense viral, bacterial or host derived DNA in the cytosol and signal via the ER resident "stimulator of IFN genes" (STING). ${ }^{23,24}$ After integration of such signaling pathways by the central kinase TBK-1, type I IFN expression is induced by transcription factors of the IRF family, namely IRF3, IRF7 or IRF5, alone or in combination. ${ }^{25}$

To resolve the signaling pathway responsible for IFN- $\beta$ induction by growing tumors, we crossed the IFN- $\beta$ reporter mouse with mice carrying deletions of different adaptor proteins or transcription factors. We then compared the reporter gene activity in tumors of such mice with the signals of reporter mice with no additional gene deletion. Mice lacking the TLR adaptors Trif, MyD88 or both, which results in a complete loss of TLR signaling, showed no alteration in the IFN- $\beta$ reporter gene activity when bearing a tumor (Fig. $4 \mathrm{~A}$ ). Therefore, none of the TLRs can be involved in the induction of type I IFN in neoplasia. Mice lacking the RLR adaptor Cardif showed a weak but significant reduction in the luciferase signal (Fig. 4 B). In contrast, lack of the adaptor protein STING drastically decreased IFN- $\beta$ gene expression in the tumor tissue (Fig. 4 B). We confirmed this finding by qPCR in an independently derived STING defective mouse strain (Fig. 4 C). This dependency is reflected in the tumor growth of MCA fibrosarcoma in $\mathrm{STING}^{-/-}$mice. Like in IFN- $\beta^{-/-}$mice ${ }^{8}$ the tumor grew significantly faster than in WT mice.

Reporter mice lacking IRF transcription factors showed that the absence of IRF7 had no impact on the bioluminescent signal (Fig. 5 A). In contrast, IRF3 deficiency strongly decreased the reporter activity in tumors. However, the signal was not completely abolished in IRF3 ${ }^{-/-}$mice suggesting a contribution of additional transcription factors. Therefore, we investigated a potential role of IRF5. Indeed, IRF5 deficiency significantly decreased the reporter signal (Fig. 5 B). Taken together, these results demonstrate that type I IFN induction in resident DCs of solid tumors is dependent on the adaptor protein STING and the transcription factors IRF3 and IRF5. 


\section{Tumor cells contribute to IFN- $\beta$ production}

The question remained whether tumor cells of the growing neoplasia would also contribute to the production of IFN- $\beta$. This would be left unnoticed by our reporter system. Therefore, we inoculated WT and IFN- $\beta^{-/-}$mice s.c. with MCA205 cells. On day 14 after tumor injection, the mice were sacrificed and IFN- $\beta$ gene expression in MCA205 tumors was assessed at the transcriptional level. As depicted in Figure 6, tumors growing in WT mice contain $\sim 10$ times more IFN- $\beta$ transcript then tumors grown in IFN- $\beta^{-/-}$mice. Therefore most of the signal is derived from the infiltrating host cells. Nonetheless in IFN- $\beta^{-/-}$mice, the detected IFN- $\beta$ transcript can only be derived from the transplanted tumor cells. Nevertheless, IFN- $\beta$ transcripts can clearly be detected in tumors from such mice. Thus, tumor cells contribute to the total IFN- $\beta$ amount in growing tumors. To validate whether IFN- $\beta$ was constitutively expressed or induced, we analyzed expression of MCA cells grown in culture. As expected, some background transcription of IFN- $\beta$ can be detected. ${ }^{26}$ However, expression in tumor cells transplanted into mice is significantly higher than in culture. This suggests induction of IFN- $\beta$ in tumor cells in vivo. 


\section{Discussion}

The importance of endogenous type I IFNs in immune surveillance of cancer is widely accepted. This is based on depletion studies using neutralizing antibodies and recombinant mice $\left(I F N A R^{-/-}\right.$, $\left.I f n b 1^{-/}\right)$. Significantly faster tumor growth of a broad variety of transplantable murine tumors is observed under such conditions. ${ }^{8,27,28}$ Tumor growth interference by type I IFN can be attributed to both, direct as well as indirect effects. As such, type I IFNs have been demonstrated to increase the level of MHC class $\mathrm{I}^{29}$ or induce growth arrest and apoptosis in cancer cells. ${ }^{30}$ Moreover, endogenous type I IFNs exert diverse immune modulatory functions, i.e. are involved in cancer immunoediting $^{31}$, stimulate innate immune cells like NK cells ${ }^{32}$ and macrophages ${ }^{33}$, promote the adaptive anti-tumor response by activating cytotoxic $\mathrm{CD}^{+} \mathrm{T}$ cells ${ }^{34}$ as well as $\mathrm{DCs}^{35}$ and enhance humoral immunity. ${ }^{36}$ Thus it is no surprise that tumors use the type I IFN system as target for immune escape. ${ }^{37}$ Despite this extensive accumulation of knowledge, many key aspects of type I IFN activities during tumor induction and progression are still unknown.

Previous studies failed to detect significant induction of IFN- $\alpha / \beta$ in various spontaneous or transplantable tumors. ${ }^{38}$ This might be due to an insufficient sensitivity of the read out. In the present work, we demonstrate the induction of type I IFN by different transplantable murine tumors using a very sensitive luciferase reporter mouse. It allows measuring IFN- $\beta$ expression by noninvasive imaging but also by ex vivo determination of the enzymatic activity of luciferase in various tissues. ${ }^{12}$ The sensitivity is comparable to a well-established PCR and by far outperforms specific ELISA. The reporter sensitivity has already been proven during Listeria monocytogenes infection, where IFN- $\beta$ was hardly measurable by ELISA while strong signals could unambiguously be detected in the reporter mice. ${ }^{16}$ Although the IFN- $\beta$ signal in our tumor situation was low, expression was clearly above the background and reached expression levels typical for constitutively produced IFN- $\beta$ in the thymus. ${ }^{12}$ 
Host cell derived type I IFNs appears to be essential in cancer surveillance. ${ }^{38}$ However, it was still not entirely clear which cells are responsible for producing this cytokine in growing tumors. Although we cannot exclude minor contributions by other host cell populations like endothelial cells, the conditional reporter mouse allowed a definition of the major producer cell type under our conditions. Accordingly, the cell type specific reporter mice revealed that IFN- $\beta$ was mainly produced by a subpopulation of tumor infiltrating DCs. This was confirmed by transcriptional analysis of isolated tumor infiltrating $\mathrm{CD} 11 \mathrm{c}^{+} \mathrm{CD} 11 \mathrm{~b}^{+} \mathrm{DC}$. In addition, the dependence on IRF5 is supporting the notion of DC being producer cells. They had been shown to depend on IRF5 for induction of TypeI IFN after viral infections. ${ }^{14}$

From the receptors responsible for induction of type I IFN under our conditions, we excluded TLRs and we found a minor contribution of RLRs. In contrast, deletion of the cytosolic DNA receptor signaling adaptor $\mathrm{STING}^{23,39}$ substantially reduced IFN- $\beta$ gene expression in tumor tissue. Thus, our results are in agreement with the observations by Woo et. al. who showed that self DNA presumably of dying tumor cells is inducing type I IFN expression in tumor tissue. ${ }^{40}$ Similarly, Deng et al. showed that radiation induces Type I IFN in tumors via STING and the DNA sensor cGAS. ${ }^{41}$

How external DNA can reach the cytosolic DNA sensing system is still not clear. Interestingly, the anti-microbial peptide LL37, which is overexpressed in psoriasis patients, very efficiently transports extracellular self-DNA into the cytosol of plasmacytoid DCs. ${ }^{42}$ Moreover, TLR independent type I IFN induction has been reported upon phagocytosis of apoptotic cells by DNase II-deficient macrophages. ${ }^{43}$ Similarly, transfer of bacterial DNA or expression plasmids from bacteria into the cytosol of antigen presenting cells (APC) has been demonstrated. ${ }^{44}$ This would suggest a general mechanism for transfer of exogenous DNA to the cytosol of APC, similar to the transfer of exogenous antigen for cross-presentation. 
Massive cell death occurs in growing tumors, either due to external assault ${ }^{41}$ or to insufficient angiogenesis and lack of oxygen. Phagocytosis of such dying tumor cells could be a potential source of DNA mediating type I IFN induction in tumor infiltrating DCs. ${ }^{20,41,43}$ On the other hand, gap junctions between tumor cells and DC have been described. ${ }^{45}$ This might be an alternative way for tumor cell DNA to reach the cytosol of DCs.

Further elaborating on the cellular source of type I IFNs in growing tumors, the present results indicate that invading host DCs are the major producers of IFN- $\beta$. However, tumor cells themselves are also induced to express IFN- $\beta$ in vivo. The signal obtained from ex vivo isolated tumor cells is significantly stronger than from cells derived from culture. Tumor cells have been shown to be capable of producing IFN after recognizing different TLR ligands. ${ }^{46}$ However, in vivo the induction of IFN- $\beta$ might also be indirect. Tumor infiltrating DCs could act as sensors. To induce IFN- $\beta$, cGAMP, the ligand of STING, produced by cGAS could be transported from the DCs to the tumor cells via gap junctions.$^{47}$ This would explain why the tumor cells themselves are induced to produce more IFN- $\beta$ in mice than in tissue culture. Experiments employing mice with an inactivated cGAS could clarify this issue. ${ }^{48}$

The importance of tumor derived type I IFN is yet unclear. In different human tumors such as glioblastoma multiforme ${ }^{49}$ or malignant melanoma ${ }^{50}$ mutations interfering with the type I IFN system have been observed. Hence, mechanisms and relevance on prognosis of tumor cell derived type I IFN should be further investigated in such cancer patients.

Interestingly, Jin and colleagues recently identified a human loss-of-function variant of STING. ${ }^{18}$ The resulting protein displays a $\geq 90 \%$ decreased potential to stimulate IFN- $\beta$ production in response to Listeria monocytogenes infection. This clearly emphasizes the importance of STING in humans. The authors assume that approximately 3\% of the American population are homozygous for this mutation. It would be very important to further investigate the impact of this 
genetic variant on tumor development, especially in the light of the present study. Unfortunately, no data on cancer associated deaths are available for this cohort far.

Taken together, the present findings regarding tumor-induced, STING-IRF3/IRF5-mediated type I IFN expression in tumor infiltrating DCs have important implications. They reveal potent molecular and cellular targets for future cancer therapy and they significantly improve our understanding of the mechanisms underlying type I IFN modulated immune surveillance of tumors.

\section{Acknowledgements}

The authors thank Susanne zur Lage and Regina Lesch for expert technical assistance. We also thank Prof. Dr. Carlos Guzman (HZI, Braunschweig, Germany) for providing C57BL/6JTmem $173^{\mathrm{gt}} / \mathrm{J}$ mice. Tie 2 cre mice were kindly provided by Prof. Dr. Bernd Arnold (DKFZ, Heidelberg, Germany). Bastian Opiz (Charité, Berlin, Germany) kindly sent us the Tmem $173^{\text {tmlCamb }}$ mice generated in the lab of John Cambier.

\section{Grant support}

This work was partly supported by the Deutsche Krebshilfe and the German Research Council (DFG). Additional support was provided by the Hannover Biomedical Research School via the Molecular Medicine PhD program and the Helmholtz Graduate School. MMB was supported by the SFB900 of the German Research Council. 


\section{References}

1. Isaacs A, Lindenmann J. Virus interference. I. The interferon. By A. Isaacs and J. Lindenmann, 1957. J Interferon Res 1987; 7:429-38.

2. Pestka $\mathrm{S}$, Krause $\mathrm{CD}$, Walter MR. Interferons, interferon-like cytokines, and their receptors. Immunol Rev 2004;202:8-32.

3. Gonzalez-Navajas JM, Lee J, David M, Raz E. Immunomodulatory functions of type I interferons. Nat Rev Immunol 2012;12:125-35.

4. Gough DJ, Messina NL, Clarke CJP, Johnstone RW, Levy DE. Constitutive type I interferon modulates homeostatic balance through tonic signaling. Immunity 2012;36:166-74.

5. Honda K, Mizutani T, Taniguchi T. Negative regulation of IFN-alpha/beta signaling by IFN regulatory factor 2 for homeostatic development of dendritic cells. Proc Natl Acad Sci U S A 2004;101:2416-21.

6. Deonarain R, Verma A, Porter AC, Gewert DR, Platanias LC, Fish EN. Critical roles for IFN-beta in lymphoid development, myelopoiesis, and tumor development: links to tumor necrosis factor alpha. Proc Natl Acad Sci U S A 2003;100:13453-8.

7. Wang BX, Rahbar R, Fish EN. Interferon: current status and future prospects in cancer therapy. $J$ Interf Cytokine Res 2011;31:545-52.

8. Jablonska J, Leschner S, Westphal K, Lienenklaus S, Weiss S. Neutrophils responsive to endogenous IFN-beta regulate tumor angiogenesis and growth in a mouse tumor model. $J$ Clin Invest 2010;120:1151-64.

9. Jablonska J, Wu C-F, Andzinski L, Leschner S, Weiss S. CXCR2-mediated tumor-associated neutrophil recruitment is regulated by IFN- $\beta$. Int J Cancer 2013;134 (6):1346-58.

10. Andzinski L, Wu C-F, Lienenklaus S, Kröger A, Weiss S, Jablonska J. Delayed apoptosis of tumor associated neutrophils in the absence of endogenous IFN- $\beta$. Int $J$ Cancer 2015;136:572-83.

11. Wu C-F, Andzinski L, Kasnitz N, Kröger A, Klawonn F, Lienenklaus S, Weiss S, Jablonska J. The lack of type I interferon induces neutrophil-mediated pre-metastatic niche formation in the mouse lung. Int J Cancer 2015;137:837-47.

12. Lienenklaus S, Cornitescu M, Zietara N, Łyszkiewicz M, Gekara N, Jabłónska J, Edenhofer F, Rajewsky K, Bruder D, Hafner M, Staeheli P, Weiss S. Novel reporter mouse reveals constitutive and inflammatory expression of IFN-beta in vivo. J Immunol 2009;183:3229-36.

13. Häcker H, Redecke V, Blagoev B, Kratchmarova I, Hsu L-C, Wang GG, Kamps MP, Raz E, Wagner H, Häcker G, Mann M, Karin M. Specificity in Toll-like receptor signalling through distinct effector functions of TRAF3 and TRAF6. Nature 2006;439:204-7.

14. Lazear HM, Lancaster A, Wilkins C, Suthar MS, Huang A, Vick SC, Clepper L, Thackray L, Brassil MM, Virgin HW, Nikolich-Zugich J, Moses A V, et al. IRF-3, IRF-5, and IRF-7 coordinately regulate the type I IFN response in myeloid dendritic cells downstream of MAVS signaling. PLoS Pathog 2013;9:e1003118.

15. Mancl ME, Hu G, Sangster-Guity N, Olshalsky SL, Hoops K, Fitzgerald-Bocarsly P, Pitha PM, Pinder K, Barnes BJ. Two discrete promoters regulate the alternatively spliced human interferon regulatory factor-5 isoforms. Multiple isoforms with distinct cell type-specific expression, localization, regulation, and function. $J$ Biol Chem 2005;280:21078-90.

16. Solodova E, Jablonska J, Weiss S, Lienenklaus S. Production of IFN- $\beta$ during Listeria monocytogenes infection is restricted to monocyte/macrophage lineage. PLoS One 2011;6:e18543.

17. Spanier J, Lienenklaus S, Paijo J, Kessler A, Borst K, Heindorf S, Baker DP, Kröger A, Weiss S, 
Detje CN, Staeheli P, Kalinke U. Concomitant TLR/RLH signaling of radioresistant and radiosensitive cells is essential for protection against vesicular stomatitis virus infection. $J$ Immunol 2014;193:3045-54.

18. Jin L, Xu L-G, Yang I V, Davidson EJ, Schwartz DA, Wurfel MM, Cambier JC. Identification and characterization of a loss-of-function human MPYS variant. Genes Immun 2011;12:263-9.

19. Ivashkiv LB, Donlin LT. Regulation of type I interferon responses. Nat Rev Immunol 2014;14:3649.

20. Fuertes MB, Kacha AK, Kline J, Woo S-R, Kranz DM, Murphy KM, Gajewski TF. Host type I IFN signals are required for antitumor CD8+ T cell responses through CD8alpha+ dendritic cells. $J$ Exp Med 2011;208:2005-16.

21. Noppert SJ, Fitzgerald KA, Hertzog PJ. The role of type I interferons in TLR responses. Immunol Cell Biol 2007;85:446-57.

22. Loo Y-M, Gale M. Immune signaling by RIG-I-like receptors. Immunity 2011;34:680-92.

23. Paludan SR, Bowie AG. Immune sensing of DNA. Immunity 2013;38:870-80.

24. Cai X, Chiu Y-H, Chen ZJ. The cGAS-cGAMP-STING pathway of cytosolic DNA sensing and signaling. Mol Cell 2014;54:289-96.

25. Barnes B, Lubyova B, Pitha PM. On the role of IRF in host defense. J Interferon Cytokine Res 2002;22:59-71.

26. Taniguchi T, Takaoka a. A weak signal for strong responses: interferon-alpha/beta revisited. Nat Rev Mol Cell Biol 2001;2:378-86.

27. Gresser I, Maury C, Bandu MT, Belardelli F. Importance of interferon alpha in the resistance of allogeneic C57B1/6 mice to the multiplication of Friend erythroleukemia cells in the liver. Int $J$ Cancer 1990;45:364-71.

28. Picaud S, Bardot B, De Maeyer E, Seif I. Enhanced tumor development in mice lacking a functional type I interferon receptor. J Interferon Cytokine Res 2002;22:457-62.

29. Maguire JE, Gresser I, Williams AH, Kielpinski GL, Colvin RB. Modulation of expression of MHC antigens in the kidneys of mice by murine interferon-alpha/beta. Transplantation 1990;49:130-4.

30. Takaoka A, Hayakawa S, Yanai H, Stoiber D, Negishi H, Kikuchi H, Sasaki S, Imai K, Shibue T, Honda K, Taniguchi T. Integration of interferon-alpha/beta signalling to p53 responses in tumour suppression and antiviral defence. Nature 2003;424:516-23.

31. Dunn GP, Bruce AT, Sheehan KCF, Shankaran V, Uppaluri R, Bui JD, Diamond MS, Koebel CM, Arthur C, White JM, Schreiber RD. A critical function for type I interferons in cancer immunoediting. Nat Immunol 2005;6:722-9.

32. Mizutani T, Neugebauer N, Putz EM, Moritz N, Simma O, Zebedin-Brandl E, Gotthardt D, Warsch W, Eckelhart E, Kantner H-P, Kalinke U, Lienenklaus S, et al. Conditional IFNAR1 ablation reveals distinct requirements of Type I IFN signaling for NK cell maturation and tumor surveillance. Oncoimmunology 2012;1:1027-37.

33. Bekisz J, Sato Y, Johnson C, Husain SR, Puri RK, Zoon KC. Immunomodulatory effects of interferons in malignancies. J Interferon Cytokine Res 2013;33:154-61.

34. Harlin H, Meng Y, Peterson AC, Zha Y, Tretiakova M, Slingluff C, McKee M, Gajewski TF. Chemokine expression in melanoma metastases associated with CD8+ T-cell recruitment. Cancer Res 2009;69:3077-85.

35. Bald T, Landsberg J, Lopez-Ramos D, Renn M, Glodde N, Jansen P, Gaffal E, Steitz J, Tolba R, Kalinke U, Limmer A, Jönsson G, et al. Immune-cell poor melanomas benefit from PD-1 blockade 
after targeted type I IFN activation. Cancer Discov 2014;

36. Le Bon A, Schiavoni G, D’Agostino G, Gresser I, Belardelli F, Tough DF. Type i interferons potently enhance humoral immunity and can promote isotype switching by stimulating dendritic cells in vivo. Immunity 2001;14:461-70.

37. Zelenay S, Van Der Veen AG, Boettcher JP, Snelgrove KJ, Rogers N, Acton SE, Chakravarty P, Girotti MR, Marais R, Quezada SA, Sahai E, Reis E Sousa C. Cyclooxygenase-Dependent Tumor Growth through Evasion of Immunity. Cell 2014;162:1257-70.

38. Gresser I, Belardelli F. Endogenous type I interferons as a defense against tumors. Cytokine Growth Factor Rev 2002;13:111-8.

39. Keating SE, Baran M, Bowie AG. Cytosolic DNA sensors regulating type I interferon induction. Trends Immunol 2011;32:574-81.

40. Woo S-R, Fuertes MB, Corrales L, Spranger S, Furdyna MJ, Leung MYK, Duggan R, Wang Y, Barber GN, Fitzgerald K a., Alegre M-L, Gajewski TF. The STING pathway mediates innate immune sensing of immunogenic tumors. Immunity 2014;41:830-42.

41. Deng L, Liang H, Xu M, Yang X, Burnette B, Arina A, Li X-D, Mauceri H, Beckett M, Darga T, Huang X, Gajewski TF, et al. STING-dependent Cytosolic DNA Sensing Promotes Radiationinduced Type I interferon-dependent Antitumor Immunity in Immunogenic Tumors. Immunity 2014;41:843-52.

42. Lande R, Gregorio J, Facchinetti V, Chatterjee B, Wang Y-H, Homey B, Cao W, Wang Y-H, Su B, Nestle FO, Zal T, Mellman I, et al. Plasmacytoid dendritic cells sense self-DNA coupled with antimicrobial peptide. Nature 2007;449:564-9.

43. Fuertes MB, Woo S-R, Burnett B, Fu Y-X, Gajewski TF. Type I interferon response and innate immune sensing of cancer. Trends Immunol 2013;34:67-73.

44. Darji A, Guzma C a, Gerstel B, Wachholz P, Timmis KN. Using Attenuated S . typhimurium. 1997;91:765-75.

45. Saccheri F, Pozzi C, Avogadri F, Barozzi S, Faretta M, Fusi P, Rescigno M. Bacteria-induced gap junctions in tumors favor antigen cross-presentation and antitumor immunity. Sci Transl Med 2010;2:44ra57.

46. Gatti G, Nuñez NG, Nocera DA, Dejager L, Libert C, Giraudo C, Maccioni M. Direct effect of dsRNA mimetics on cancer cells induces endogenous IFN- $\beta$ production capable of improving dendritic cell function. Eur J Immunol 2013;43:1849-61.

47. Ablasser A, Schmid-Burgk JL, Hemmerling I, Horvath GL, Schmidt T, Latz E, Hornung V. Cell intrinsic immunity spreads to bystander cells via the intercellular transfer of cGAMP. Nature 2013;503:530-4.

48. Schoggins JW, MacDuff DA, Imanaka N, Gainey MD, Shrestha B, Eitson JL, Mar KB, Richardson RB, Ratushny A V, Litvak V, Dabelic R, Manicassamy B, et al. Pan-viral specificity of IFNinduced genes reveals new roles for cGAS in innate immunity. Nature 2014;505:691-5.

49. Sgorbissa A, Tomasella A, Potu H, Manini I, Brancolini C. Type I IFNs signaling and apoptosis resistance in glioblastoma cells. Apoptosis 2011;16:1229-44.

50. Culig Z. Suppressors of cytokine signalling-3 and -1 in human carcinogenesis. Front Biosci (Schol Ed) $2013 ; 5: 277-83$. 
Figure legends

Figure 1. IFN- $\beta$ induction in transplantable murine tumors. (a) Tumors cells of 4T1, CT26, MCA205 or LLC1 were injected s.c. into the back of IFN- $\beta$ reporter mice. Luciferase reporter activity was assessed directly before inoculation and every 24 hours after inoculation. Depicted are the results obtained before tumor cell inoculation and 24 hours, 72 hours as well as 14 days after tumor inoculation. (b) Quantification of the light signal in a region of interest surrounding the site of tumor cell injection (4T1: $n=5 ;$ CT26: $n=6$; MCA205: $n=8$; LLC1: $n=5$ ). Statistical significances were calculated using one way ANOVA with turkey post-test $(* \mathrm{p} \leq 0,05 ; * * \mathrm{p} \leq$ $0,01 ; * * * \mathrm{p} \leq 0,001)$

Figure 2. Ex vivo luciferase assay for IFN- $\beta$ induction in different tumors and organs. On day 14 after s.c. inoculation of MCA205 or CT26 tumor cells into IFN- $\beta$ reporter mice on $\mathrm{C} 57 \mathrm{BL} / 6$ or $\mathrm{BALB} / \mathrm{c}$ background, respectively, reporter activity was assessed ex vivo. The relative luciferase units (RLU) in homogenates were normalized on tissue weight.

Figure 3. Definition of IFN- $\boldsymbol{\beta}$ producing host cells infiltrating tumors. To assess the cellular source of IFN- $\beta$ in tumors, global IFN- $\beta$ reporter mice as well as reporter mice specific for (a) Tie2 (endothelial/hematopoietic cells), (b) LysM (myeloid lineage cells), (c) CD11c (dendritic cells), (d) CD4 (T cells and subpopulation of DCs) and (e) CD19 (B cells) expressing cells were injected with MCA205 tumor cells. On day 14 after tumor inoculation, the mice were sacrificed and ex vivo luciferase measurement was performed. Statistical significance was calculated using unpaired students t test $(* * \mathrm{p} \leq 0,005, * * * \mathrm{p} \leq 0,0005)$. (f) To validate tumor infiltrating DCs as 
cellular source of IFN- $\beta$ in the tumor microenvironment, CD11 $\mathrm{c}^{+} \mathrm{CD} 11 \mathrm{~b}^{-} \mathrm{DC}, \mathrm{CD} 11 \mathrm{c}^{+} \mathrm{CD}_{11 \mathrm{~b}^{+}}$ DCs and $\mathrm{CD}_{19}{ }^{+} \mathrm{B}$ cells were sorted from tumor and spleen 14 days after tumor inoculation. Quantitative RT-PCR analysis of IFN- $\beta$ gene expression was performed. Data represent the mean of at least five pooled animals. The experiment was confirmed once.

Figure 4. Signaling pathways involved in type I IFN induction in tumor infiltrating host cells. MCA205 fibrosarcoma were inoculated s.c. into the back of WT reporter animals as well as reporter animals deficient for (a) MyD88, Trif or MyD88 and Trif or (b) Cardif and STING $\left(\mathrm{MPYS}^{-/}\right)$. On day 14 after tumor cell injection, the mice were sacrificed and ex vivo luciferase measurement was performed. (c) The role of STING was confirmed using qRT-PCR for IFN- $\beta$ gene expression in total tumor tissue of C57BL/6 wild type mice or STING deficient mice $\left(\right.$ Tmem $173^{\mathrm{g} / \mathrm{t}}$ ). Two independent STING deficient mouse strains were used in (b) and (c). The data represent a mean of at least 4 animals per group. (d) Tumor growth curve of MCA205 fibrosarcoma in WT and $\mathrm{STING}^{\mathrm{gt}}$ mice. Statistical significances were calculated using unpaired students $t$ test or one way ANOVA with turkey post-test $(* \mathrm{p} \leq 0,05)$.

Figure 5. IRFs involved in type I IFN induction in tumors. MCA205 fibrosarcoma cells were inoculated s.c. into the back of global IFN- $\beta$ reporter animals as well as reporter animals that were deficient for the IRF family members (a) IRF3 or IRF7 or (b) IRF5. The data represent a mean of at least 4 animals per group. Statistical significances were calculated using unpaired students $t$ test or one way ANOVA with turkey post-test $\left({ }^{*} p \leq 0,05\right)$. 
Figure 6. Tumor infiltrating host cells as well as tumor cells produce IFN- $\beta$ in vivo. On day 14 after s.c. inoculation of MCA205 fibrosarcoma cells into the back of C57BL/6 wild type and syngeneic IFN- $\beta^{-/-}$animals, total RNA from tumor tissue was harvested. After reverse transcription, qPCR analysis of IFN- $\beta$ gene expression in total tumor was performed. MCA205 cells from cell culture were included as controls. Statistical significance was calculated using unpaired students t test $(* \mathrm{p} \leq 0,05, * * \mathrm{p} \leq 0,005, * * * \mathrm{p} \leq 0,0005)$. 
a

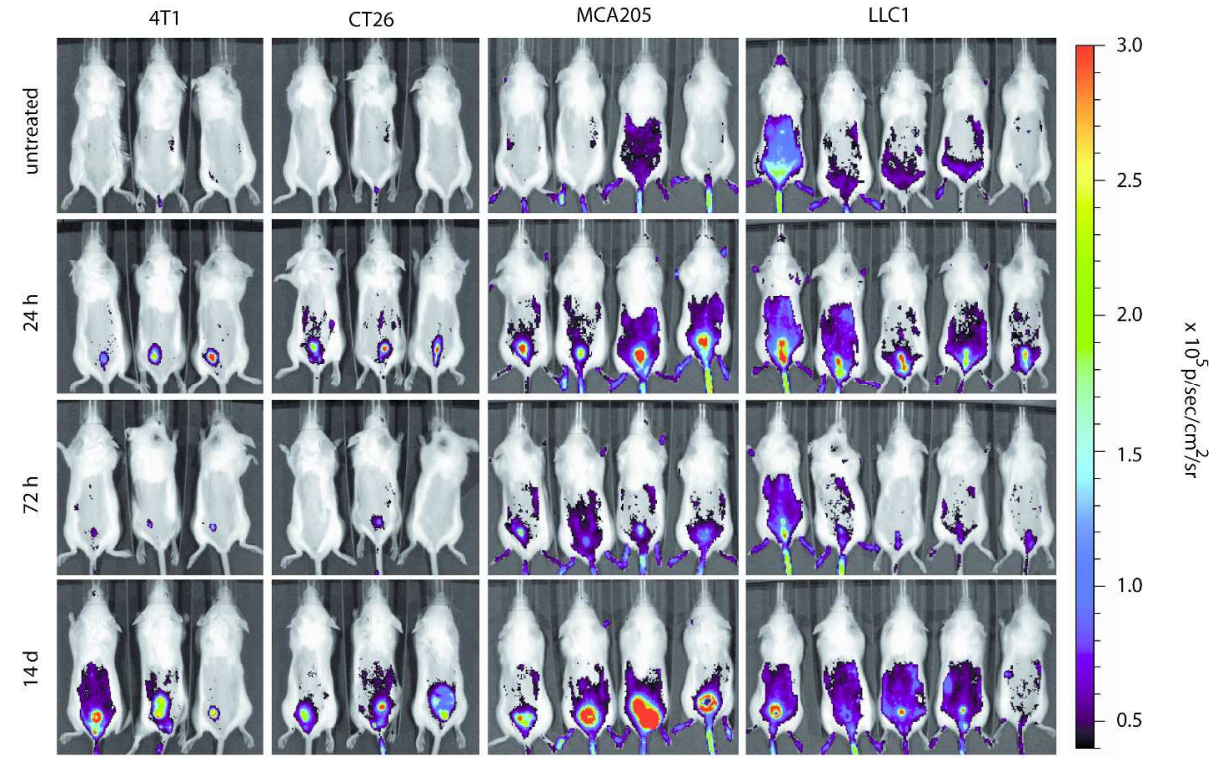

b
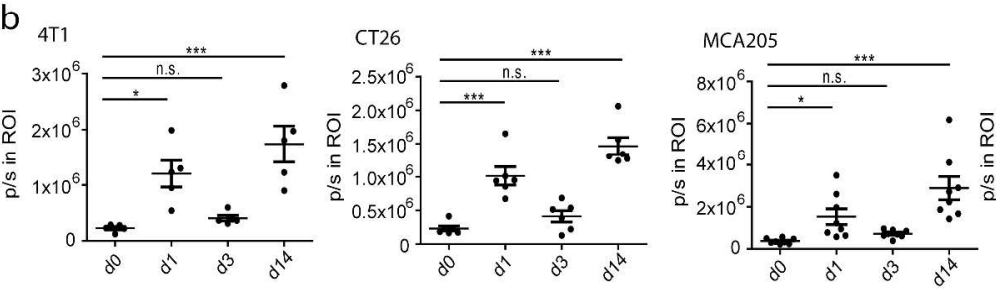

LLC1

Figure 1. IFN- $\beta$ induction in transplantable murine tumors. (a) Tumors cells of 4T1, CT26, MCA205 or LLC1 were injected s.c. into the back of IFN- $\beta$ reporter mice. Luciferase reporter activity was assessed directly before inoculation and every 24 hours after inoculation. Depicted are the results obtained before tumor cell inoculation and 24 hours, 72 hours as well as 14 days after tumor inoculation. (b) Quantification of the light signal in a region of interest surrounding the site of tumor cell injection (4T1: $n=5 ; C T 26: n=6 ; M C A 205$ : $\mathrm{n}=8$; LLC1: $\mathrm{n}=5$ ). Statistical significances were calculated using one way ANOVA with turkey post-test

$$
(* \mathrm{p} \leq 0,05 ; * * \mathrm{p} \leq 0,01 ; * * * \mathrm{p} \leq 0,001) \text {. }
$$




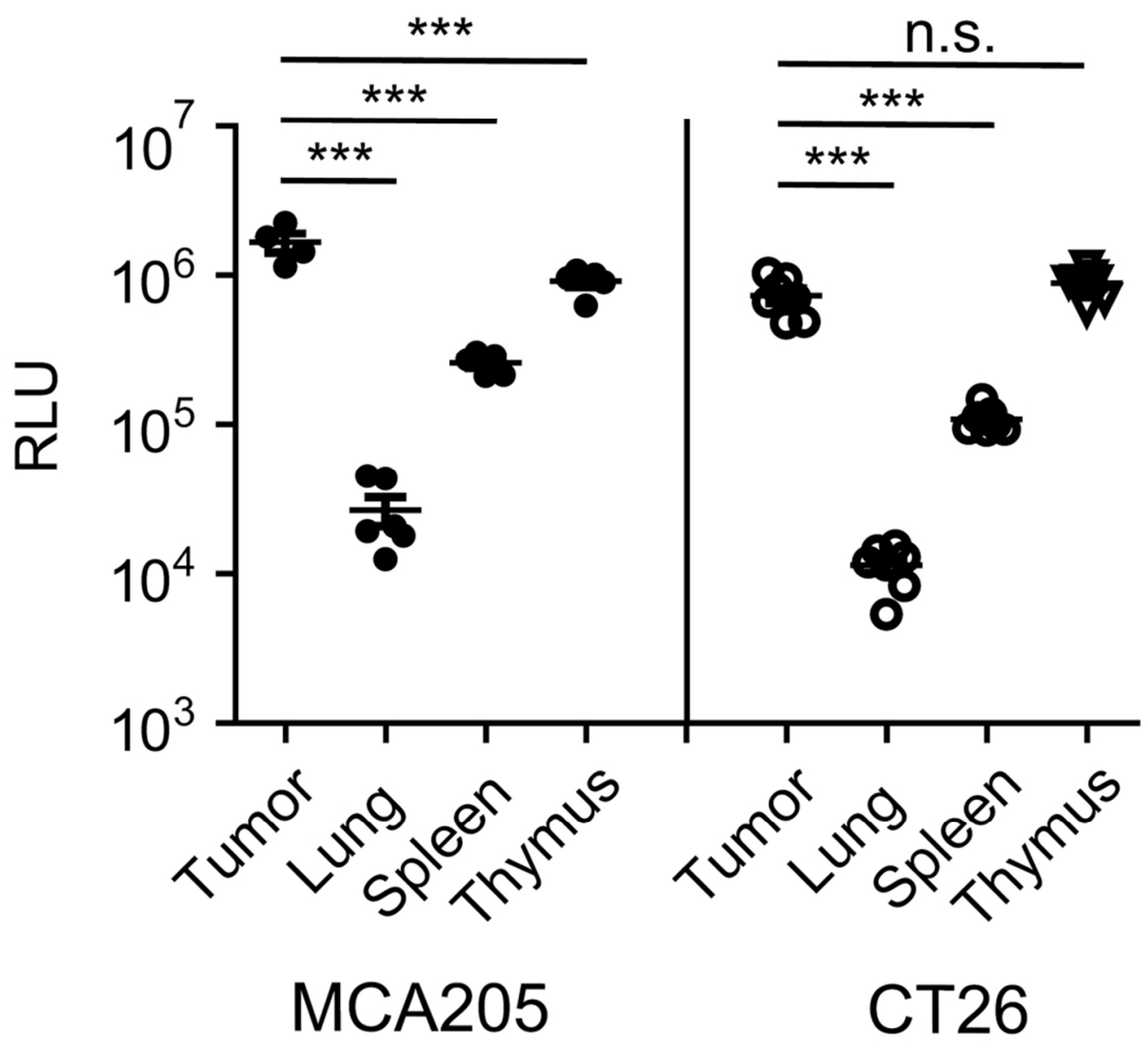

Figure 2. Ex vivo luciferase assay for IFN- $\beta$ induction in different tumors and organs. On day 14 after s.C. inoculation of MCA205 or CT26 tumor cells into IFN- $\beta$ reporter mice on C57BL/6 or BALB/c background, respectively, reporter activity was assessed ex vivo. The relative luciferase units (RLU) in homogenates were normalized on tissue weight. $88 \times 82 \mathrm{~mm}(300 \times 300 \mathrm{DPI})$ 

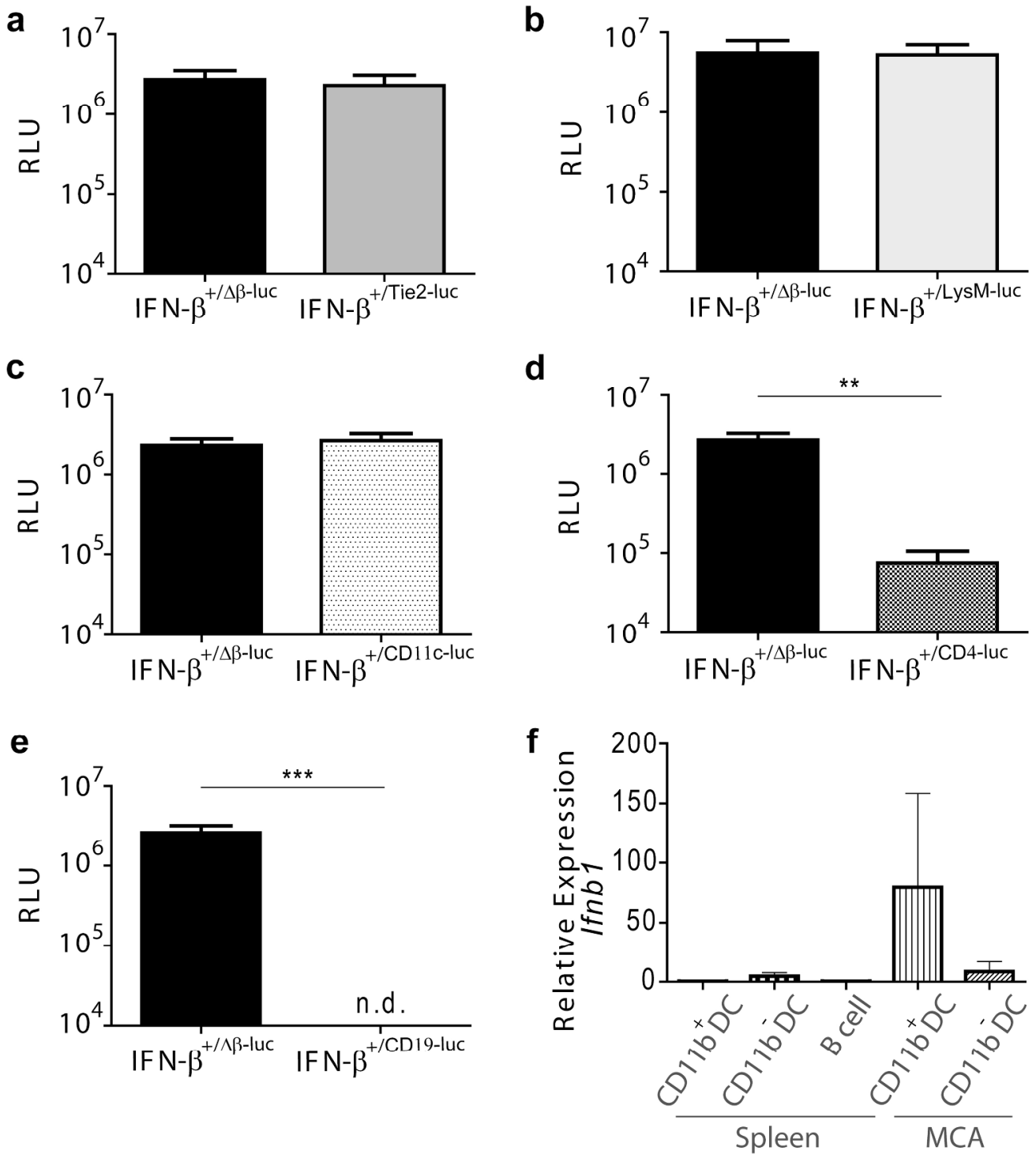

Figure 3. Definition of IFN- $\beta$ producing host cells infiltrating tumors. To assess the cellular source of IFN- $\beta$ in tumors, global IFN- $\beta$ reporter mice as well as reporter mice specific for (a) Tie2 (endothelial/hematopoietic cells), (b) LysM (myeloid lineage cells), (c) CD11c (dendritic cells), (d) CD4 (T cells and subpopulation of DCs) and (e) CD19 (B cells) expressing cells were injected with MCA205 tumor cells. On day 14 after tumor inoculation, the mice were sacrificed and ex vivo luciferase measurement was performed. Statistical significance was calculated using unpaired students t test ( $* * p \leq 0,005, * * * p \leq 0,0005$ ). (f) To validate tumor infiltrating DCs as cellular source of IFN- $\beta$ in the tumor microenvironment, CD11C+ CD11b- DC,

CD11c+ CD11b+ DCs and CD19+ B cells were sorted from tumor and spleen 14 days after tumor inoculation. Quantitative RT-PCR analysis of IFN- $\beta$ gene expression was performed. Data represent the mean of at least five pooled animals. The experiment was confirmed once. 
a

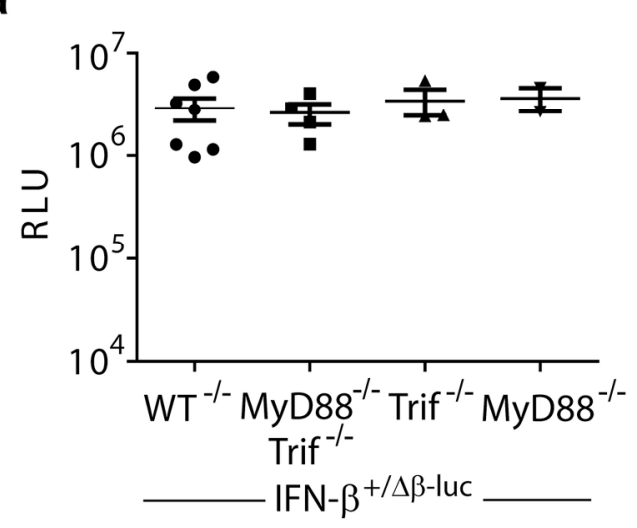

C

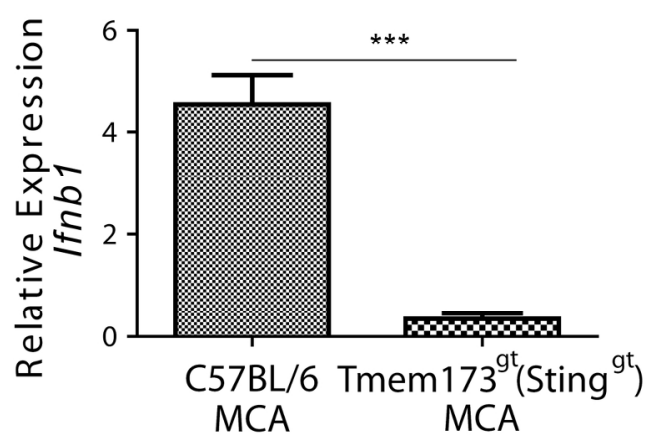

b

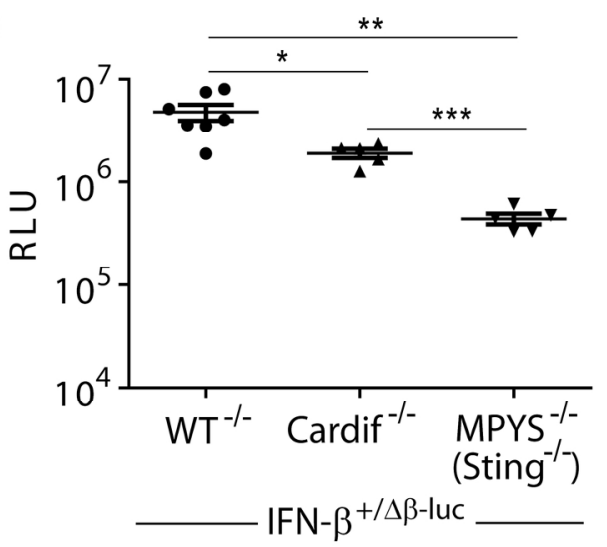

d

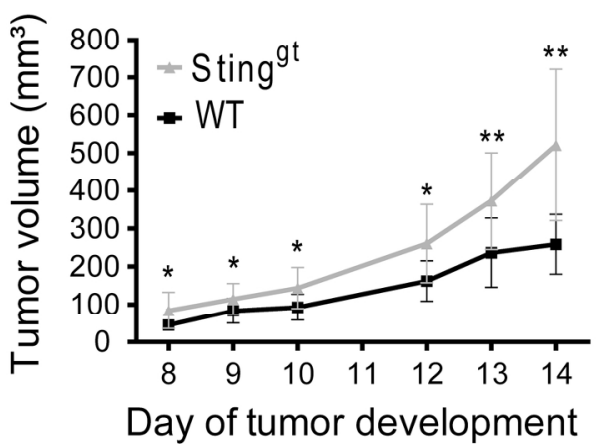

Figure 4. Signaling pathways involved in type I IFN induction in tumor infiltrating host cells. MCA205 fibrosarcoma were inoculated s.c. into the back of WT reporter animals as well as reporter animals deficient for (a) MyD88, Trif or MyD88 and Trif or (b) Cardif and STING (MPYS-/-). On day 14 after tumor cell injection, the mice were sacrificed and ex vivo luciferase measurement was performed. (c) The role of STING was confirmed using qRT-PCR for IFN- $\beta$ gene expression in total tumor tissue of C57BL/ 6 wild type mice or STING deficient mice (Tmem173g/t). Two independent STING deficient mouse strains were used in (b) and (b). The data represent a mean of at least 4 animals per group. (d) Tumor growth curve of MCA205 fibrosarcoma in WT and STINGgt mice. Statistical significances were calculated using unpaired students $\mathrm{t}$ test or one way ANOVA with turkey post-test $(* p \leq 0,05)$. 
a

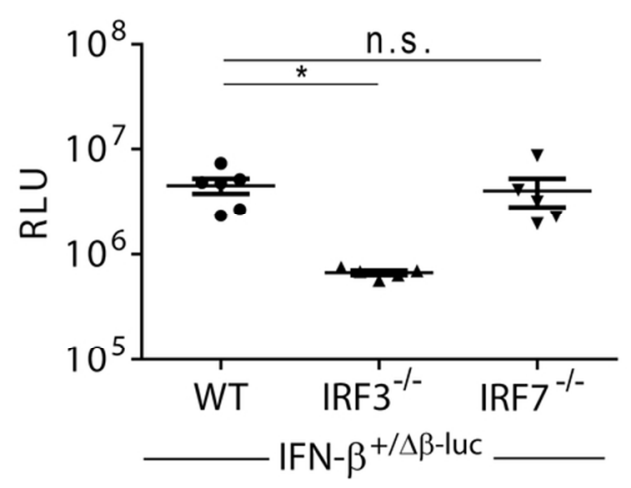

b

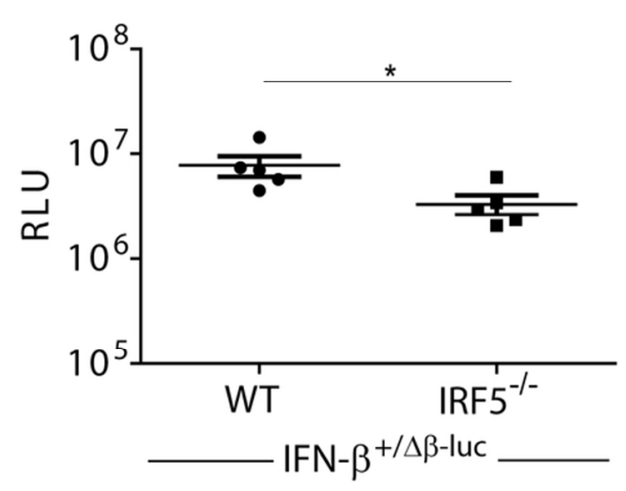

Figure 5. IRFs involved in type I IFN induction in tumors. MCA205 fibrosarcoma cells were inoculated s.c. into the back of global IFN- $\beta$ reporter animals as well as reporter animals that were deficient for the IRF family members (a) IRF3 or IRF7 or (b) IRF5. The data represent a mean of at least 4 animals per group. Statistical significances were calculated using unpaired students test or one way ANOVA with turkey posttest $(* p \leq 0,05)$.

$85 \times 35 \mathrm{~mm}(300 \times 300$ DPI $)$ 


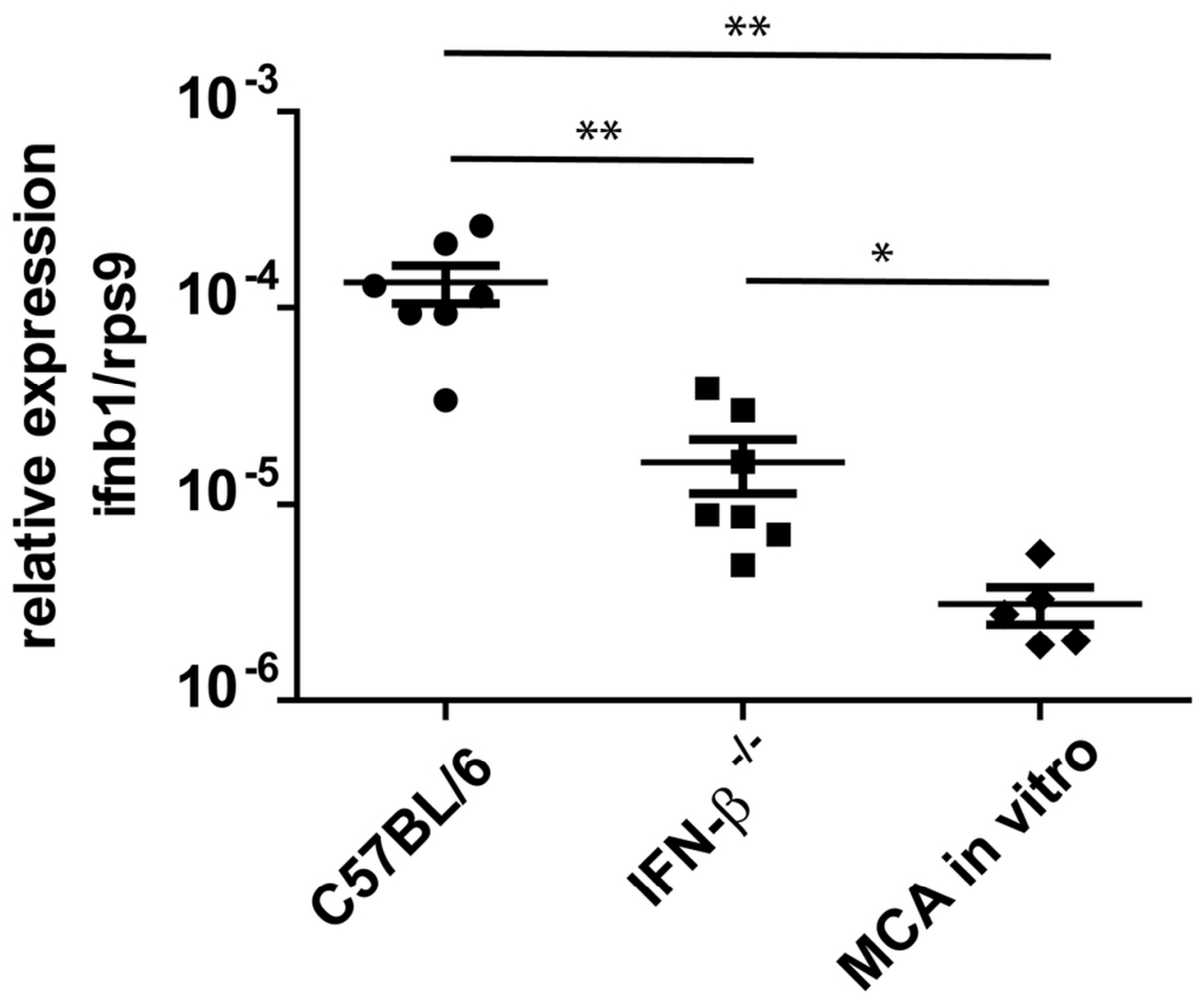

Figure 6. Tumor infiltrating host cells as well as tumor cells produce IFN- $\beta$ in vivo. On day 14 after s.c. inoculation of MCA205 fibrosarcoma cells into the back of C57BL/ 6 wild type and syngeneic IFN- $\beta-/-$ animals, total RNA from tumor tissue was harvested. After reverse transcription, qPCR analysis of IFN- $\beta$ gene expression in total tumor was performed. MCA205 cells from cell culture were included as controls. Statistical significance was calculated using unpaired students test $(* p \leq 0,05, * * p \leq 0,005, * * * p \leq$ $0,0005)$.

$85 \times 72 \mathrm{~mm}(300 \times 300 \mathrm{DPI})$ 